\title{
An Adjustment Method for the Customized Trains of the Railway
}

\author{
Xiaojuan Li $\mathbb{D}^{1},{ }^{1}$ Jiana Shi, ${ }^{1}$ Li Yang, ${ }^{2}$ Hongwei Wang, ${ }^{1}$ and Miao Yan ${ }^{1}$ \\ ${ }^{1}$ Transportation Institute, Inner Mongolia Engineering Research Center for Urban Transportation Data Science and Applications, \\ Inner Mongolia University, Zhao-Jun-Road \#24, Hohhot 010070, Inner Mongolia, China \\ ${ }^{2}$ Passenger Transport Department, China Railway Hohhot Group Co., Ltd., Hohhot 010010, Inner Mongolia, China \\ Correspondence should be addressed to Xiaojuan Li; 311987431@imu.edu.cn
}

Received 30 July 2019; Accepted 30 December 2019; Published 1 February 2020

Academic Editor: Roberta Di Pace

Copyright (c) 2020 Xiaojuan Li et al. This is an open access article distributed under the Creative Commons Attribution License, which permits unrestricted use, distribution, and reproduction in any medium, provided the original work is properly cited.

\begin{abstract}
The "customized train" is a train operated by the customized service of the passengers. The departure time, total travel time, and the travel classes are decided by passengers' wishes. Successful operation of customized trains (CT) has a great significance to the future development in the relationship between the railway enterprise and the passenger service. In order to establish a comprehensive method for the CT operation plan, the paper proposes an intention survey method to obtain the passenger travel wishes data. Then, according to the existing timetable, the alternative set of CTs is built. The CT should satisfy the constraints of the passenger demand, the stop, and the train travel time. Based on the alternative set, a bilevel programming model for the maximum operation revenue of the CTs and minimum adjustment cost of existing timetable is established according to the passengers' travel demands. The model also considers the constraints of actual travel time, arrival headway, departure headway, maximal adjustment time and capacity, and so on. The adjustment strategies for the train operation confliction are established. Finally, the method is proven through the analysis of actual cases, which can provide decision-making basis for future railway enterprises when operating the customized trains.
\end{abstract}

\section{Introduction}

In recent years, many service enterprises are constantly accelerating the pace of reform and renewal to meet the growing demand of the passengers. In many service-oriented areas, service providers started to care more about improvement of the service quality rather than the capacity increase. To deal with such challenges, the traditional railway transportation industries are attempting reform on customized services. That is why customized trains (CTs) are introduced. However, because of the development of social economy and technology, there are significant changes in transportation market in terms of service concept, which make the problem of maximizing operation benefit and satisfying passengers' travel demand extremely complex and hard to be solved using traditional operation modes. In China, Xi'an Railway enterprise has successfully operated the first customized train. The passenger attendance factor of this train reached $100 \%$, which shows that CT is accepted by the passengers.
But, so far, there are not many researches on operation adjustment method of CT. Gallo et al [1] proposed the transit network design problems under the assumption of elastic demand. D'Acierno et al. [2] analysed the interaction between travel demand and railway capacity. D'Acierno et al. [3] analysed effects on travel demand of different levels of degraded services in the case of metro system failure in order to define the best strategy to adopt so as to minimize user discomfort. CTs can satisfy the passengers' travel demands including the departure time, total travel time, and the travel class. Some literatures had studied the interaction between travel demand and railway services.

The CTs have changed the relationship mode of the railway enterprise and the passengers. In this new customized mode of train operation, passengers can choose the time period they want to travel and the seat according to their own wishes. Railway enterprise operates the trains to meet the passenger's travel demands based on the passenger travel wishes data. These data can be obtained by the survey. At the same time, CTs can expand the effective supply, which 
embodies the service concept of people-oriented service in railway industry. It is a major step of the transformation from supply-oriented to demand-oriented.

Therefore, the main innovations of the paper include the following:

(1) Demand of the CTs was obtained by the questionnaires. The operation of CT is based on the prediction of passenger demand. Therefore, the reasonable demand acquisition can better provide services for passengers. This paper uses the intention survey to collect the data of passengers' travel intention for the CTs. This method can obtain the data of departure time, travel time, and travel class needed by passengers in customized service. The obtained data can provide theoretical data support for the departure time and train marshalling plan

(2) The method for the alternative CT is built. There are some uncertainties in the operation of CTs, which need to be constrained by various conditions. There will be a variety of operation schemes of the CTs; different schemes will adapt to different operation scenarios. Therefore, it is very important to establish the alternative set for CTs. In this paper, the alternative set is established by considering the constraints such as passenger demand, train stops, and the train operation time. The alternative customized trains can be obtained, which meet both operation conditions and passenger travel demand

(3) A bilevel programming model of train operation adjustment for the CTs is built. The model can consider both customized demand of passengers and revenue of railway enterprises. Finally, a reasonable solution solved by the model can be provided for the customized train service

\section{Literature Review}

There are two parts of the literatures for the paper: one is the customized service and the other is the train operation adjustment.

2.1. Customized Service. With the significant increases in customer demands, personalized demands play an increasingly important role in current services. Feng et al. [4] proposed a four-level customized-service framework, and a service intention model was presented and discussed.

For the area of the traffic and transportation, the existing literatures mainly studied the problems of the customized bus. Liu and Ceder [5] defined the customized bus, that is, the service that can provide advanced, personalized, and flexible demand-responsive public transport to specific clientele, especially commuters. Ma et al. [6] proposed a method for the stop selection, line planning, and timetable of the customized buses based on the passenger travel data. The authors proposed a route selection model based on the demand characteristics and operational purpose for the customized bus. This model considered the operating cost and social benefits, and a branch-and-bound-based solution method was developed. Cao and Wang [7] proposed the method of passenger assignment on customized buses that could provide the benefits to passengers by balancing the elements of travel time, waiting time, delay, and economic cost. Tong et al. [8] developed a joint optimization model for the customized bus service. The model optimized the loading rate requirements for the bus operators to reach long-term and detailed bus routing and timetabling plans. The method could make a flexible decision for matching passengers to bus routes. Zhou et al. [9] developed an analytical model to select a public transport service provision between park and ride and on-demand public bus (ODPB). The model could maximize the total social welfare, which includes consumer surplus and operator's net profit. Meng et al. [10] studied the scheduling method of multicustomized bus lines with passenger travel time window constraints. An optimization model of customized bus dispatching is established, which aims at minimizing the total operating mileage of multiple buses. The impact of passenger departure point and destination on the model solution was analysed.

In addition, for the customized service for the transportation, there are some related research problems that have been studied.

\subsubsection{Demand Responsive Transport (DRT) Problem.} Brake et al. [11] proposed that the UK has achieved initial success in implementing DRT. They mainly focused on some elements such as service characteristics (flexibility of route selection, flexibility of booking method, and prebooking time), emerging markets, and the overall contribution of DRT to increased social inclusion. Cich et al. [12] studied the demand response transmission (DRT) simulation. A simulation model was constructed to assess the impact of the cooperative trip sequence composer on the feasibility of demand-responsive transport service providers with the minimum operating costs. Haverkamp [13] first applied DRT for current heavy rail services. This paper focused on the problem of how do network structure and passenger demand distribution relate to station platform capacity, track capacity, fleet size, level of service, and offered seatkilometers in rail DRT systems.

2.1.2. Dial-A-Ride Problem. CTs are used to determine the route, time, and stop of the travel by collecting passengers' willingness to travel, which is similar to dial-a-ride transport. The dial-a-ride transport is divided into two kinds: door-todoor dial-a-ride transit (DDDART) and checkpoint dial-aride transit (CPDART). Based on consideration of the range of service travelers, this paper focuses on the CPDART.

Daganzo [14] set up some simplifying assumptions to compare the total operating costs (the sum of operating cost and travel time cost) of the three service modes: FRT, DDDART, and CPDART. It was proposed that CPDART was better than FRT for choosing the stop more flexibly of the bus. Pratelli [15] elaborated some modelling methods for CPDART problem, including the analytical method, the heuristic method, and the mathematical programming 
method. Some of the most recent mathematical methods to deal with the deviation bus route design problem had been described.

At present, there are not sufficient researches on CTs in the world. A few CTs have been put into practice. Zhang and Dong [16] proposed the method of increasing trains in the existing timetable. The integer programming model is constructed to solve the problem of maximum number of the trains on the timetable. Meanwhile, the constraints of departure time, headway, dwell time, capacity, and the latest arrival time were set. This method can be referenced for increasing the CTs on the existing timetable. According to the method of customized bus and the optimal adjustment method of the timetable, we propose an optimization method for CT operation. We built the bilevel optimization model by considering the benefit railway enterprise and the influence of the existing timetable based on the passengers demand. The train's stop plan and the total travel time are considered simultaneously.

2.2. Adjustment Method of the Train Operation. For the adjustment method of the train operation, the problem of the train movement is important. Lulli et al. [17] and Oneto et al. [18] studied four important aspects of the train movements in the larger-scale railway network: the travel time, the dwell time, the train delay, and the penalty costs. The European International Railway Union [19] described railway capacity by timetable compression method, which provides a classic approach for the train adjustment. Espinosa-Aranda et al. [20] and Corman et al. [21] studied the train operation adjustment model by minimizing the average delay time of all passengers. Some constraints of passengers' travel service were considered. Other literatures built the adjustment model and algorithm by minimizing the number or delay time of the trains [22-24]. Our objective function of the adjustment model is the minimal adjustment time of the trains at each stop station. The number of the adjustment trains is considered as the main constraint.

Goverde [25, 26] and $\mathrm{Li}$ et al. [27] studied the train operation adjustment method using the Max-Plus algebra. Then, Li et al. [28] proposed six adjustment strategies on train operation adjustment to minimize the total delay time of the trains at each station. Zhan et al. [22], Zhou et al. [29], and Ghaemi et al. [30] also proposed some effective adjustment strategies. These strategies mainly included acceleration, the delayed waiting of trains in the station, moving operation times, exchanging operation order, and changing stop plan.

Some adjustment strategies are utilized by this paper, including (1) acceleration in the section, (2) exchanging the train operation order by increasing the overtaking, and (3) the adjusted trains being postponed according to the minimum headway.

\section{Problem Statement}

We propose a comprehensive method for the CT selection and adjustment. This method can be divided into three stages: demand acquisition, CT scheme selection, and train operation adjustment on the existing timetable.

For the first stage, based on the questionnaires by passengers, the expected departure time period, the total travel time, and the travel class demand of the passengers can be obtained. The passengers would be reduced when the actual departure time of the CT is not in the expected departure time period. Therefore, the rate of the reduced passengers for different time period between the actual departure time and the expected departure time period can be collected. These data can provide a basis for the operation plan and stop plan of the CTs.

For the second stage, based on passenger travel wishes data and the existing timetable, a comprehensive method for the CT selection and adjustment is established. The alternative set of CTs is a group of CTs that can meet passenger service demand and transport capacity simultaneously. When a selected train satisfies the constraints of the passenger demand, the stop, and the train operation time, the train can be added in the alternative set of CTs. Then a variety of CT operation schemes are formulated based on the alternative set. The scheme considers many factors, such as the stop plan, the number of CTs, train marshalling plan, the travel time, and the arrival time.

For the third stage, a bilevel programming model of train operation adjustment is built to implement the comprehensive optimization for enterprise operation benefit and passengers' travel service demands. On the upper-level model, the decision-maker is the railway enterprise, and the model's objective is to maximize the revenue. The goal of lower-level model is to minimize the adjustment cost of existing trains. Then, three main adjustment strategies are proposed to resolve the train conflict.

Then, the method of this paper has some research premises as follows.

(1) The method in the paper mainly applies to the railway lines within the jurisdiction of a railway company. For the long distance railway line, the operation of CTs needs to coordinate with multiple railway companies. This condition will affect the train operation of multiple lines in the network, so it is difficult to organize and implement. For the short-distance railway line, the travel time of the train is short, and the departure frequency is generally high, so passengers do not need the customized services. Therefore, the method in the paper is mainly applied to the medium-long-distance $(4-8 \mathrm{~h})$ railway service within the jurisdiction of a railway company

(2) There is a potentially infinite number of trains available in the origin stations. Also, there is a potentially infinite number of tracks available in the destination stations. D'Acierno et al. [31] provided an analytical approach for defining operational parameters for metro systems by considering these assumptions, such as reserve time. Therefore, these assumptions can ensure that the customized train can operate on the basis of the existing timetable 
(3) The dwell times is set a priori and it is independent of the passengers' behavior and number. Some researches had studied the dwell time in the metro railway. D'Acierno et al. [32] calculated dwell times as a function of travel demand flows evaluated by simulating explicitly user behavior on the platform when a train arrives. Wang and Yin [33] defined that the passenger travel model in urban rail platform has four parts: the enter passenger walking, the passenger waiting distribution, passenger boarding, and alighting passengers walking. But Botte and D'Acierno [34] considered the dwell times set as the fixed values would result in deviations between actual and planned operations. Because the CTs operation plan is determined before the train running, it is difficult to determine the passengers' behavior and number in each station. The dwell times is set as a fixed value

\section{Models}

The paper synthetically considers the operation revenue of railway enterprise and adjustment cost of the existing trains based on the passenger demand. A bilevel programming model is built to realize the comprehensive optimization.

The parameters in the paper are depicted in Table 1.

The trains in the alternative set of CTs can operate in the line that meets the passenger demands and capacity simultaneously. The attributes of the trains include the original station, terminal station, train operation route, train speed, marshalling plan, and stop plan.

Then, the constraints for the alternative CTs can be expressed as follows:

(1) Constraint of the passenger demand

The seat capacity of the CT should not be less than the actual passenger demands, which can be expressed by the following equation:

$$
\left[i=1 \sum^{n_{c}}\left(n_{i, w} \cdot N_{w}+n_{i . z} \cdot N\right)\right] \cdot R_{o} \geq D
$$

(2) Constraint of the stop

When the passenger flow of a station is not less than a certain value- $d_{\text {min }}$, the CTs need to add a stop at this station. The constraint can be expressed by equation (2), where $X_{i, s}$ is a binary value. When train $i$ stops at station $s$, the value of $X_{i, s}$ is 1 ; otherwise, the value of $X_{i, s}$ is 0 .

$$
X_{i, s}= \begin{cases}0, & \left(d_{s s^{\prime}}<d_{\min }\right), \\ 1, & \left(d_{s s^{\prime}} \geq d_{\min }\right), \\ \forall i, s . & \end{cases}
$$

\section{(3) Constraint of train travel time}

For this constraint, we consider the problem in two aspects: one is that the original departure time of the

\begin{tabular}{|c|c|}
\hline Parameters & Definitions \\
\hline$n_{c}$ & Number of CTs, and each train can be expressed by $i$ \\
\hline$n_{e}$ & $\begin{array}{c}\text { Number of existing trains, and each train can be } \\
\text { expressed by } j\end{array}$ \\
\hline$m$ & $\begin{array}{c}\text { Number of the stations, and each station can be } \\
\text { expressed by } s\end{array}$ \\
\hline$n_{i, w}, n_{i, z}$ & $\begin{array}{l}\text { Number of the sleeping train units/seat train units of } \\
\text { train } i\end{array}$ \\
\hline$N_{w}, N_{z}$ & $\begin{array}{c}\text { The seat capacity of one sleeping train unit/seat train } \\
\text { unit }\end{array}$ \\
\hline$R_{o}$ & Average passenger attendance factor of the CTs \\
\hline$D$ & The total passenger demand of the CTs \\
\hline$d_{s s^{\prime}}$ & The passenger demand of OD-ss' \\
\hline$t_{i, s}^{a}, t_{i, s}^{a^{\prime}}$ & $\begin{array}{c}\text { The arrival time of train } i \text { at station } s \text { before/after } \\
\text { adjustment }\end{array}$ \\
\hline$t_{i, s}^{d}, t_{i, s}^{d^{\prime}}$ & $\begin{array}{c}\text { The departure time of train } i \text { at station } s \text { before/after } \\
\text { adjustment }\end{array}$ \\
\hline$t_{i, s}^{\text {stop }}$ & The dwell time of train $i$ at station $s$ \\
\hline$t_{\max }^{\text {total }}$ & The maximal travel time of the passengers expected \\
\hline$p_{r}$ & $\begin{array}{c}\text { The proportion of the passenger demand in the time } \\
\text { period } r\end{array}$ \\
\hline$p_{s s^{\prime}}^{w}, p_{s s^{\prime}}^{z}$ & $\begin{array}{l}\text { The price of the sleeping train unit/seat train unit of } \\
\text { OD-ss }\end{array}$ \\
\hline$d_{s s^{\prime}}^{w}, d_{s s^{\prime}}^{z}$ & $\begin{array}{l}\text { The demand of the sleeping train unit/seat train unit } \\
\text { of OD-ss' }\end{array}$ \\
\hline$C_{\mathrm{op}}$ & The operation cost of one train unit per minute \\
\hline$C_{z}$ & The cost coefficient for increasing one train \\
\hline$C_{\text {adj }}$ & The adjustment cost of the existing trains \\
\hline$t_{i, s, s+1}^{y}$ & $\begin{array}{l}\text { The travel time of the trains between stations } s \text { and } \\
\qquad s+1\end{array}$ \\
\hline$t_{\text {add }}^{\mathrm{Q}}, t_{\text {add }}^{T}$ & $\begin{array}{c}\text { The additional time of departure and additional time } \\
\text { of arrival }\end{array}$ \\
\hline$I_{s}^{a}, I_{s}^{d}$ & $\begin{array}{l}\text { The arrival headway and departure headway at } \\
\text { station } s\end{array}$ \\
\hline$t_{\text {adj }}^{z}$ & The maximum adjustment time of the CTs \\
\hline$t_{i, s}^{\text {stop-min }}$ & The minimum dwell time of train $i$ at station $s$ \\
\hline$t_{i, s}^{\text {stop-max }}$ & The maximum dwell time of train $i$ at station $s$ \\
\hline$C_{s}$ & $\begin{array}{l}\text { The maximum usable capacity of station } s \text { besides the } \\
\text { existing trains }\end{array}$ \\
\hline$t_{\text {adj }}^{J}$ & The maximum adjustment time of the existing trains \\
\hline$N_{\max }$ & The maximum number of adjusted trains \\
\hline
\end{tabular}

TABle 1: Parameters definition.

CT cannot affect the trains in the existing timetable, as shown in equation (3), and the other is that the total travel time of the CTs should not be more than the maximum travel time required by passengers, which can be expressed by equation (4).

$$
\begin{gathered}
t_{i, s}^{d} \neq t_{i^{\prime}, s}^{d}, \quad \forall i, j, s ; i \neq i^{\prime}, \\
\sum_{s=1}^{m}\left[\left(t_{i, s+1}^{a}-t_{i, s}^{d}\right)+X_{i, s} t_{i, s}^{\text {stop }}\right] \leq t_{\text {max }}^{\text {total }} .
\end{gathered}
$$

4.1. Upper-Level Model. The decision-maker of the upperlevel model is the railway enterprise, which pursues the maximum benefit when operating the CT. Therefore, the objective function of the model is to maximize the total 
revenue of the $\mathrm{CT}$ as shown in equation (5). The part before the minus sign represents the total ticket revenue of the passengers and another part expresses the CT operation costs, where $\lambda(t)$ is the function for the relationship of the passenger demand and the time difference between the actual operation time and passenger's preferred time period.

$$
\begin{aligned}
\max Z_{1}= & \sum_{s=1}^{m} \sum_{s^{\prime}=1}^{m} \sum_{r=1}^{n r}\left[\lambda(t) p_{r}\left(P_{s s^{\prime}}^{w} d_{s s^{\prime}}^{w}+P_{s s^{\prime}}^{z} d_{s s^{\prime}}^{z}\right)\right] \\
& -C_{z} n_{c} \sum_{i=1}^{n_{c}} \sum_{s=1}^{m}\left\{C _ { o p } ( n _ { i , z } + n _ { i , w } ) \left[\left(t_{i, s+1}^{a}-t_{i, s}^{d}\right)\right.\right. \\
& \left.\left.+X_{i, s} t_{i, s}^{\text {stop }}\right]\right\},
\end{aligned}
$$

s.t.

$$
\begin{aligned}
& t_{i, s+1}^{a}-t_{i, s}^{d} \geq t_{i, s, s+1}^{y}+X_{i, s+1} t_{\mathrm{add}}^{T}+X_{i, s} t_{\mathrm{add}}^{Q}, \\
& t_{i+1, s}^{a}-t_{i, s}^{a} \geq I_{s}^{a}, \\
& t_{i+1, s}^{d}-t_{i, s}^{d} \geq I_{s}^{d}, \\
& \left|t_{i, s}^{a^{\prime}}-t_{i, s}^{a}\right| \leq t_{\mathrm{adj}}^{z}, \\
& \left|t_{i, s}^{d^{\prime}}-t_{i, s}^{d}\right| \leq t_{\mathrm{adj}}^{z}, \\
& t_{i, s}^{\mathrm{stop}-\min } \leq t_{i, s}^{d}-t_{i, s}^{a} \leq t_{i, s}^{\mathrm{stop}-\max }, \\
& \sum_{i=1}^{n} X_{i, s} \leq C_{s}, \quad \forall s .
\end{aligned}
$$

Among the previous equations, equation (6) indicates that the actual travel time between each of the sections should not be less than the minimum travel time. Equation (7) expresses that the arrival interval time of the adjacent trains should not be less than the minimum arrival headway; and then the departure interval time of the adjacent trains should not be less than the minimum departure headway, which can be expressed by equation (8); the constraints of the adjustment arrival/departure time of the CT should not be more than the maximum adjustment time, which can be expressed by equations (9) and (10). Equation (11) shows the constraints on the dwell time range of CT at any station. Equation (12) indicates that the capacity cannot be more than the maximum capacity for each station.

4.2. Lower-Level Model. Because the adjustment of the trains has a great impact on the existing timetable, this will involve train operation plan in both the line and the station, and so forth. At the same time, the train adjustment may affect the operation of the trains in the whole railway network. Therefore, the impact on existing trains should be considered while increasing the CTs. The main objective of the lower-level model is to minimize adjustment cost of the existing train line. The objective function is shown in the following equation:

$$
\min Z_{2}=C_{\mathrm{adj}}\left(\sum_{j=1}^{n_{e}} \sum_{s=1}^{m}\left|t_{j, s}^{d^{\prime}}-t_{j, s}^{d}\right|+\sum_{j=1}^{n_{e}} \sum_{s=1}^{m}\left|t_{j, s}^{a^{\prime}}-t_{j, s}^{a}\right|\right),
$$

so that

$$
\begin{gathered}
\left|t_{j, s}^{a^{\prime}}-t_{j, s}^{a}\right|<t_{\text {adj }}^{J}, \\
\left|t_{j, s}^{d}-t_{j, s}^{d^{\prime}}\right|<t_{\text {adj }}^{J}, \\
\sum_{j=1}^{n_{e}} x_{j} \leq N_{\max }, \\
x_{j}= \begin{cases}0, & \left|t_{j, s}^{d^{\prime}}-t_{j, s}^{d}\right|=0 \text { and }\left|t_{j, s}^{a^{\prime}}-t_{j, s}^{a}\right|=0, \\
1, & \left|t_{j, s}^{d^{\prime}}-t_{j, s}^{d}\right|>0 \text { or }\left|t_{j, s}^{a^{\prime}}-t_{j, s}^{a}\right|>0, \\
\forall j, s . & \end{cases}
\end{gathered}
$$

Among the previous equations, equation (14) indicates that the adjustment time difference of train arrival time cannot exceed the maximum adjustment time. Equation (15) indicates that the adjustment time difference of train departure time cannot exceed the maximum adjustment time. Equation (16) shows that the number of adjusted existing trains cannot exceed the maximum number. Equation (17) is binary variable, indicating whether the existing train $j$ is affected and needs to be adjusted $\left(x_{j}=1\right.$ indicates that the train $j$ had been affected).

\section{The Adjustment Algorithm}

5.1. Adjustment Strategy for the Train Conflict. In [28], the train adjustment strategy has been comprehensively analysed. We choose some suitable and effective strategies to apply in this paper.

5.1.1. Section Acceleration. This adjustment strategy can be expressed by the trains acceleration on the section by compressing the buffer time. This strategy should ensure that the running time of the adjusted train in the section does not exceed the minimum running time. Therefore, the section acceleration scheme can be selected based on equation (18), which indicates that the arrival headway between the adjusted train $i$ and the front train $i-1$ should not be less than the minimum arrival headway at station $s$.

$$
t_{i, s}^{a}-t_{i, s-1}^{a} \geq t_{i, s-1, s^{\prime}}^{y} .
$$

In addition, the time can be reduced for train $i$ in the section (s-1, s) as calculated using equation (19). The time can be calculated by the minimum value between the minimum arrival headway of station $s$ and the buffer time of the train operation in section $(s-1, s)$.

$$
t_{s-1, s}^{\mathrm{acc}, i}=\min \left\{I_{s}^{a},\left[\left(t_{i, s}^{a^{\prime}}-t_{i, s}^{a}\right)-t_{i, s, s+1}^{y}\right]\right\} .
$$


5.1.2. Increase in Overtaking. As shown in Figure 1, when train $i$ has a long delay at station $s$, which is satisfied by equation (20), train $i$ can be overtaken by train $i+1$ to decrease the delay time of the latter one.

$$
t_{i+1, s}^{d^{\prime}} \leq t_{i, s}^{d^{\prime}}
$$

The constraint conditions of this strategy include station $s$ which can handle the overtaking, and overtaking between the two trains should satisfy the constraint given by equation (21). The number of the overtaking trains $n_{h}$ is determined by the maximum dwell time of the overtaken train, where $h$ is the train overtaking train $i$.

$$
I_{s}^{a}+\sum_{h=1}^{n_{h}}\left[\left(t_{h, s}^{d^{\prime}}-t_{h-1, s}^{d^{\prime}}\right)+t_{h, s}^{\text {stop-min }}+I_{s}^{d}\right] \leq t_{i, s}^{\text {stop-max }}
$$

5.1.3. Train Postponement. As shown in Figure 2, the train postponement based on the minimum headway can be selected. The delayed trains can be postponed in order as scheduled by the timetable as the minimum headway and this scheme is constrained by equation (22) (Algorithm 1).

$$
\begin{gathered}
t_{i+1, s}^{a^{\prime}}=t_{i, s}^{a^{\prime}}+I_{s}^{a} \\
\text { or } t_{i+1, s}^{d^{\prime}}=t_{i, s}^{d^{\prime}}+I_{s}^{d} .
\end{gathered}
$$

\section{Case Study}

6.1. The Alternative Set of CTs. The paper chooses the line of Hohhot to Wuhai in China. Currently there are 14 existing trains from Hohhot to Wuhai every day, with an average travel time of 6 hours. The stations in this line include Chasu Qi Station, Sala Qi Station, Baotou East Station, Baotou Station, Wulat Qianqi Station, Wuyuan Station, Linhe Station, and Bayangol Station, with a total distance of $560 \mathrm{~km}$. Wuhai City is in the southwest of Hohhot City. A large number of students and migrant workers return home during the peak period of holidays, which causes considerable increase in the number of passengers on this line. During this period, tickets have often been sold out a month before the train operation. Therefore, the CTs can solve this problem by satisfying passenger with better service in terms of the departure time, the travel time, and the travel class selection etc.

In order to obtain the data of passenger's real travel demand willingness as the data source of this paper's case analysis, this paper conducts a questionnaire survey on some passengers of 14 trains between Hohhot and Wuhai by means of field investigation and collects 1215 valid questionnaires. In the questionnaire survey, two questions are mainly investigated. One is the time period when passengers prefer to travel for 6 hours. The other is the proportion of passengers who still choose to travel by CT when there is a certain time difference between the actual CT departure time and the expected departure time. The stop plan of the CT is Hohhot-Sala Qi-Baotou East-Baotou-Linhe-BayangolWuhai.

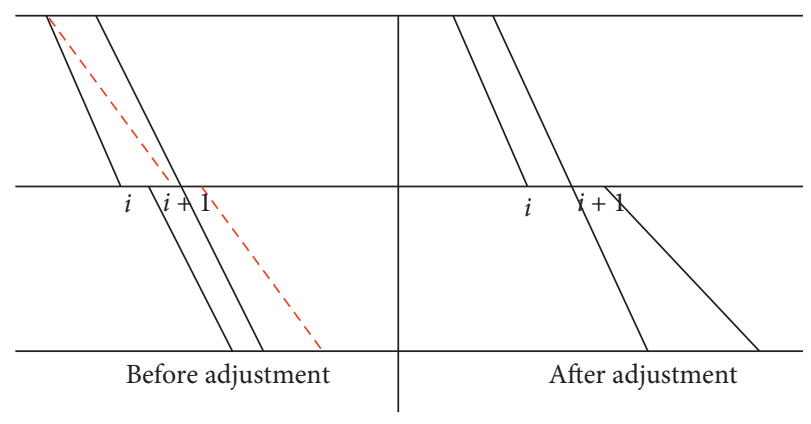

FIGURE 1: Increasing the overtaking.

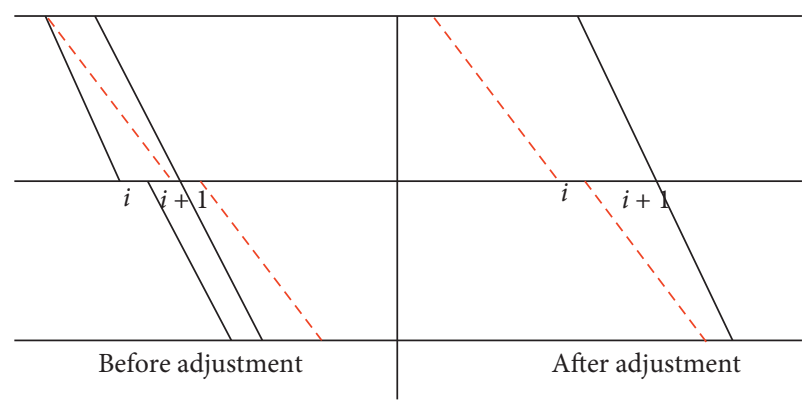

FIgURE 2: Train postponement.

In the first part of the questionnaire, the questionnaire questions are set as follows: "If the travel time is six hours, please choose the time period you expect to travel in the following travel time period." Combining with the established train operation options, the questionnaire questions are set up as follows: (7:00-10:00), (10:00-13:00), (13: 00-16:00), and (16:00 and later), four time periods for passengers to choose from. According to the data analysis, the proportion of passengers' departure time intention in each departure period is shown in Table 2.

We assume that the CT will lose part of the passengers who are not in the expected travel time period. But if the time difference between the actual departure time of CT and the expected departure time remains within a certain range, it will still attract some passengers who are expected to travel in the other time periods. In the second part of the questionnaire, the question is the following: "If a CT runs $0.5 \mathrm{~h} /$ $1 \mathrm{~h} / 1.5 \mathrm{~h} / 2 \mathrm{~h} / 2.5 \mathrm{~h} / 3 \mathrm{~h} / 3.5 \mathrm{~h} / 4 \mathrm{~h} / 4.5 \mathrm{~h} / 5 \mathrm{~h} / 5.5 \mathrm{~h} / 6 \mathrm{~h}$ before (after) the expected departure time, are you still willing to take this train?" According to the questionnaire data, the number of passengers who will still choose the CT when the actual time of CTs differs from the expected time by a certain time is obtained and the time $t=0 \mathrm{~h}, 0.5 \mathrm{~h}, 1 \mathrm{~h}, 1.5,2 \mathrm{~h}, 2.5 \mathrm{~h}$, $3 \mathrm{~h}, 3.5 \mathrm{~h}, 4 \mathrm{~h}, 4.5 \mathrm{~h}, 5 \mathrm{~h}, 5.5 \mathrm{~h}$, and $6 \mathrm{~h}$, as shown in Figure 3. An empirical function is obtained to explain the relationship between these two indications and can be expressed by $\lambda(t)=e^{-0.342 t}$.

6.2. Results and Discussion. In this scenario, the average train speed is $100 \mathrm{~km} / \mathrm{h}$, the additional time of departure and time of the train at station are $1 \mathrm{~min}$, and the minimum original departure interval time of CT is $20 \mathrm{~min}$. The minimum 
Step1: Built the alternative set of CT

Step1.1: Determine the number of CTs and the number and the ratio of sleeping train units and seat train units of each train according to equation (1);

Step1.2: Determine the stop plan of the CT according to equation (2);

Step1.3: Determine the original departure time period and the passenger demand of the CTs based on the questionnaires.

Step1.4: The alternatives set of CTs is built $\mathbf{B L}=\left\{i \in\left[1, n_{c}\right]\right\}$;

Step2: The function of CTs selection

(1) The CTs operation scheme set $\mathbf{S L}=\left\{f \in\left[1, n_{s}\right]\right\}$;

(2) $Z=0$; flag $=0 ; A=f$;

(3) For $f=1$ to $n_{s}$ do

(4) For $i=1$ to $n_{c}$ do

(5) The $\lambda(t)$ can be obtained by the questionnaires;

(6) For $s=1$ to $m$ do

(7) If equations (6)-(12) cannot be satisfied then

(8) $\quad$ flag $=1$;

(9) $Z_{1}=Z_{1}+$ Equation (5);

(10) End If

(11) End For

(12) End For

(13) If flag $==0$

(14) $Z_{2}=$ function $2(f)$;

(15) End If

(16) If $Z_{2} !=$ Null then

(17) $\quad Z^{\prime}=Z_{1}-Z_{2}$;

(18) If $Z^{\prime}>Z$ then

(19) $Z=Z^{\prime} ; A=f$;

(20) End If

(21) End If

(22) End For

Step3: The function of the train adjustment $\mathrm{Z}_{2}=$ function2 (A)

(1) $\mathrm{Nadj}=$ number of delayed trains, including the CTs.

(2) For $i=1$ to Nadj do

(3) For $s=1$ to $m$ do

(4) If $t_{i, s}^{a^{\prime}} !=t_{i, s}^{a}$ or $t_{i, s}^{d^{\prime}} !=t_{i, s}^{d}$ then

(5) $\quad \operatorname{Total}_{\mathrm{adj}}=100000$;

(6) For $u=1$ to 3 do

(7) If the constraints of the strategy $u$ is satisfied then

(8) If Tu $<$ Total $_{\text {adj }}$ Then

(9) $\quad \operatorname{Total}_{\mathrm{adj}}=T_{\mathrm{u}}$;

(10)

(12)

(13)

(14)

(15)

(16)

(17)

(18)

(19) End if

(20) End for

(21) Return $Z_{2}=$ Equation (13);

Algorithm 1

TABle 2: Proportion of passengers' departure time intention in each time period.

\begin{tabular}{lcccc}
\hline Departure time period & $7: 00-10: 00$ & $10: 00-13: 00$ & $13: 00-16: 00$ & $16: 00$ and later \\
\hline Proportion of passengers & 0.41 & 0.33 & 0.15 & 0.11 \\
\hline
\end{tabular}

headway of the intermediate station is 10 minutes. The minimum stop time and the maximal stop time are $3 \mathrm{~min}$ and $30 \mathrm{~min}$, respectively. The minimum adjustment time for both the CTs and existing trains is $20 \mathrm{~min}$. The number of the train units of the CTs is 16 , of which the ratio of sleeping train unit to seat train unit is $1: 3$. The calculation results 


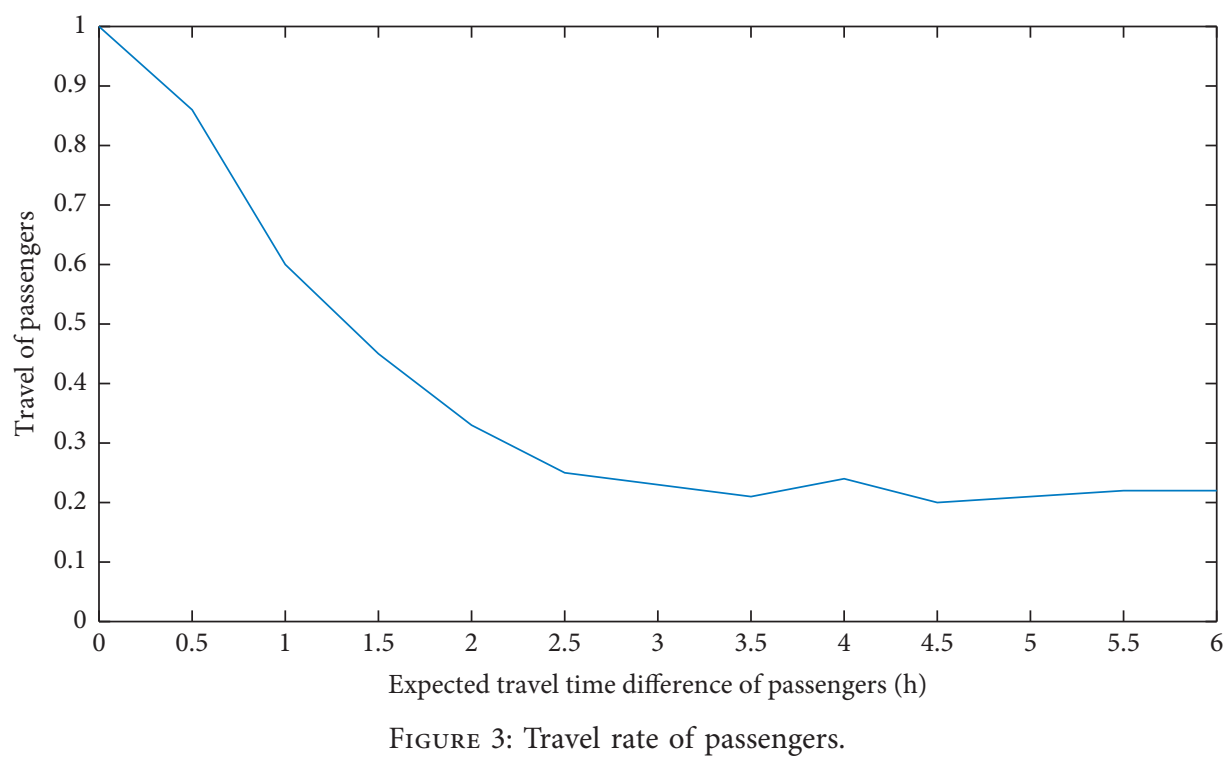

show that the reasonable departure times of CTs are as follows: train 1, $7: 10$ departure at Hohhot East, train 2, $7: 30$ departure at Hohhot East, train 3, 9:00 departure at Hohhot East, train 4, 11:00 departure at Hohhot East, train 5, $12: 10$ departure at Hohhot East, and train 6, 13:30 departure at Hohhot East, respectively. In Figure 4, the black line represents the existing train, the red line represents the CT, and the dotted line represents the train before the adjustment.

The number of train units for each train cannot be less than 5 . Therefore, the number of trains that can be operated cannot be more than 3. For the 6 CTs, 41 operation schemes can be obtained. We analyse 18 operation schemes with more optimal results in this case. These CTs operation schemes are shown in Table 3.

For CTs, some factors need to be considered, such as ratio of attracted passengers, train operation cost, and the adjustment cost on existing trains. When $C_{z}=1$ and $C_{\text {adj }}=100$, the ratio of attracted passengers of each scheme is shown in Figure 5. It can be shown that more trains operating in different periods can obviously attract passenger flow. But, at the same time, the cost of train operation also increases with the increasing of the CTs. The operation cost of CT and adjustment cost of existing lines for each scheme are shown in Figure 6.

The main costs of the CTs include train operation cost and adjustment cost of existing lines. Because these costs are difficult to quantify, the paper adopts different numerical range methods to analyse them separately.

\subsubsection{The Influence of the Operation Cost Coefficient of CTs.} By comparing the final target values of 18 schemes of CT operation, which can be selected by enterprises under different train operation cost coefficients, the changing trend of target values of different schemes can be obtained, as shown in Figure 7.

Set $C_{z}=1,1.5,2,2.5,3$, respectively, to be calculated. As shown in Figure 7, when the coefficient of train operation cost changes from 1 to 3 , the target values of the models under the different train operation schemes are given. As shown in Figure 7, it can be concluded that the target value of the model decreases with the increase of train operation cost coefficient.

When choosing one-train scheme, scheme \#4 (train 4) can obtain the maximum target value, because this train will not affect the existing train and attract the largest passenger demand. When choosing two-train scheme, the maximum target value will be obtained by scheme \#9 (train $2+$ train 5 ), because this scheme can attract more passengers and produce less adjustment cost. When choosing three-train scheme, scheme \#14 (train $2+$ train $4+$ train 6) is the optimal scheme.

Regardless of the train operation cost coefficient, scheme \#9 (train 2+train 5) has the largest target value when the trains departure at $7: 30$ and 12:10, which can attract more passengers. The target value of train operation cost and the cost generated by existing trains are lower.

The 16 train units are grouped into one CT in schemes $\# 1 \# 6$; the fluctuation range of the target value caused by the change of train operation cost coefficient is small. The 16 train units are grouped into three CTs in schemes \#12-\#18; the fluctuation range of the target value caused by the change of train operation cost coefficient is larger. When train operation cost coefficient is low, schemes \#-\#11, where 16 train units are grouped into two CTs, enterprises will obtain a larger target value. When train operation cost coefficient is large, schemes \#1\#6 will obtain a larger target value.

6.2.2. The Influence of Adjustment Cost Coefficient of Existing Train. Because CT is operated based on the existing timetable, the CT will have an impact on the existing trains. The adjustment cost coefficient of the existing trains needs to be considered. The adjustment cost coefficient of the existing trains is different in different cities, stations, and time periods. Based on the fixed operation cost coefficient of CT, the paper analyses the target values of different schemes under different adjustment cost coefficients of existing trains. 


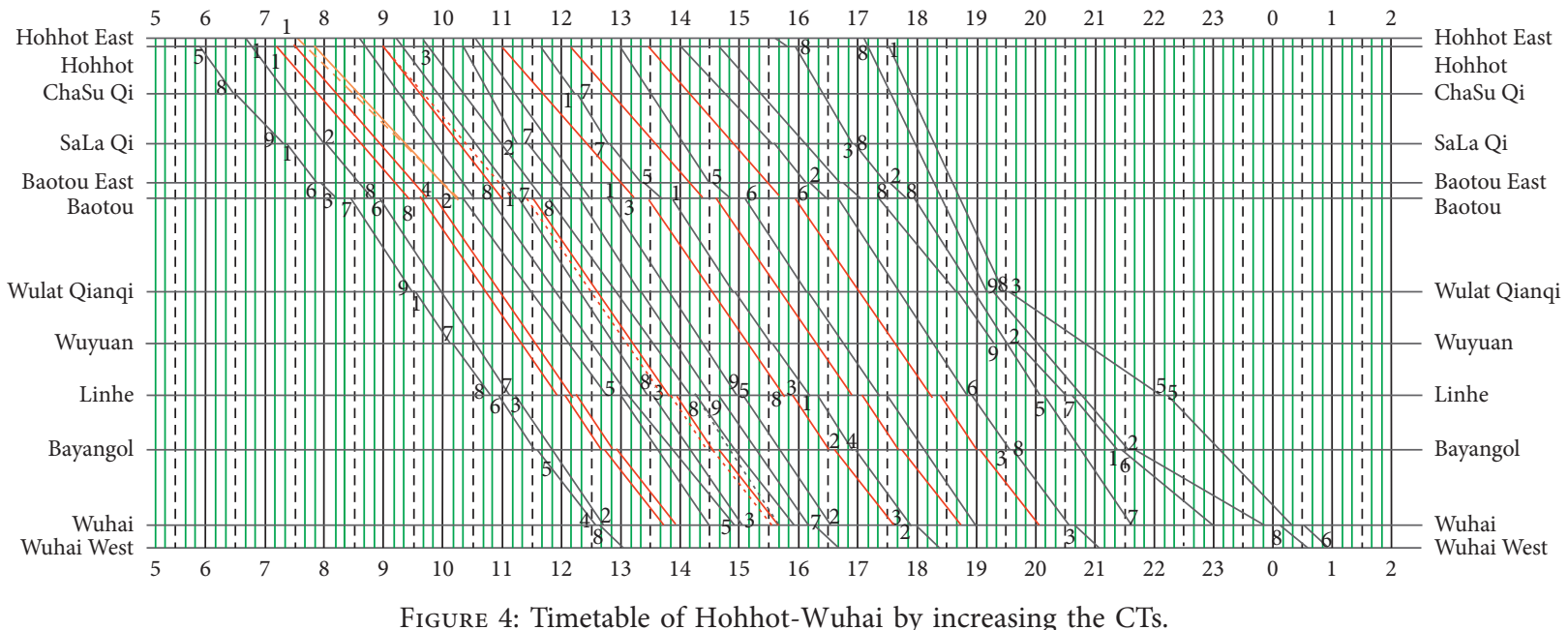

TABLE 3: operation plans of CTs.

\begin{tabular}{|c|c|c|c|c|c|c|c|}
\hline No. & Train number & Train combination & Marshalling plan & No. & Train number & Train combination & Marshalling plan \\
\hline 1 & 1 & Train 1 & 16 & 10 & 2 & Train $3+$ train 5 & $8+8$ \\
\hline 2 & 1 & Train 2 & 16 & 11 & 2 & Train $4+$ train 5 & $8+8$ \\
\hline 3 & 1 & Train 3 & 16 & 12 & 3 & Train $2+$ train $4+$ train 5 & $5+5+6$ \\
\hline 4 & 1 & Train 4 & 16 & 13 & 3 & Train $3+$ train $4+$ train 5 & $5+5+6$ \\
\hline 5 & 1 & Train 5 & 16 & 14 & 3 & Train $2+$ train $4+$ train 6 & $5+5+6$ \\
\hline 6 & 1 & Train 6 & 16 & 15 & 3 & Train $3+$ train $4+$ train 6 & $5+5+6$ \\
\hline 7 & 2 & Train $2+\operatorname{train} 4$ & $8+8$ & 16 & 3 & Train $4+$ train $5+$ train 6 & $5+5+6$ \\
\hline 8 & 2 & Train $3+$ train 4 & $8+8$ & 17 & 3 & Train $2+$ train $3+$ train 4 & $5+5+6$ \\
\hline 9 & 2 & Train $2+$ train 5 & $8+8$ & 18 & 3 & Train $1+$ train $3+$ train 4 & $5+5+6$ \\
\hline
\end{tabular}

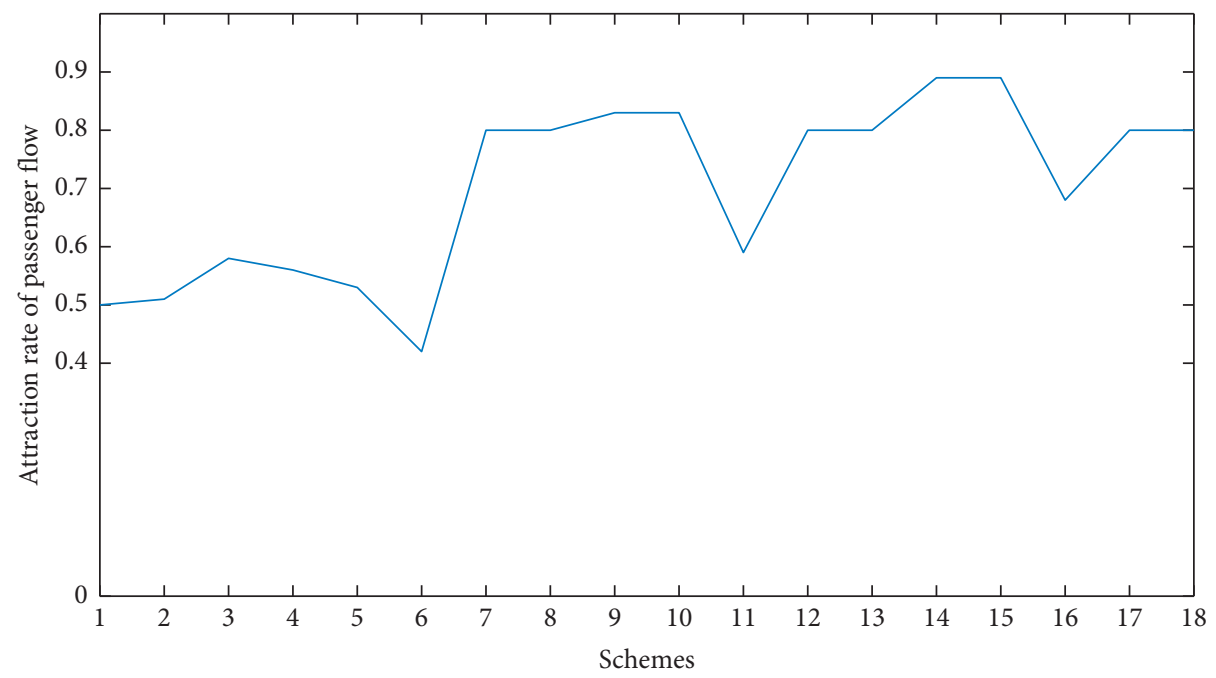

Figure 5: Attraction rate of passenger flow.

If the train operation cost coefficient $C_{z}=1$, the influence of different train adjustment cost coefficient $C_{\text {adj }}$ on each scheme is shown in Figure 8.

Regardless of the adjustment cost coefficient of the existing trains, the target value of scheme \#9 (train $2+$ train 5 ) is the greatest. Combining Figures 4 and 5, scheme \#9 can attract more passengers and the adjustment cost coefficient of existing trains is relatively less.
When the adjustment cost coefficient changes from 200 to 1000 , the target values of schemes \#4, \#16, \#5, \#11, \#1, and \#6 remain unchanged, which indicates that the CT operation in these schemes will not affect the existing trains. The target values of schemes \#9, \#14, \#7, and \#12 fluctuate slightly, which indicates that the adjustment cost coefficient of existing trains caused by CT operation in these schemes is insignificant, and CT operation has less influence on existing trains. The target 


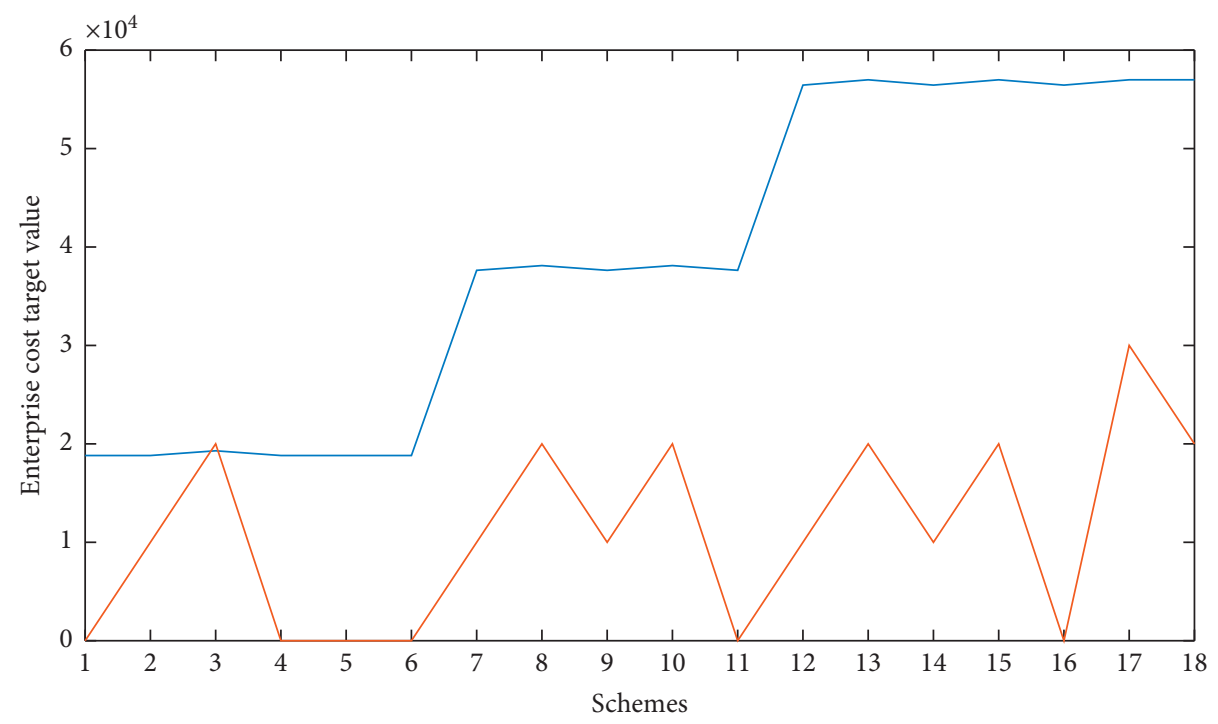

- Cost of increasing train operation

_ Cost of adjusting existing trains

Figure 6: Costs of each scheme.

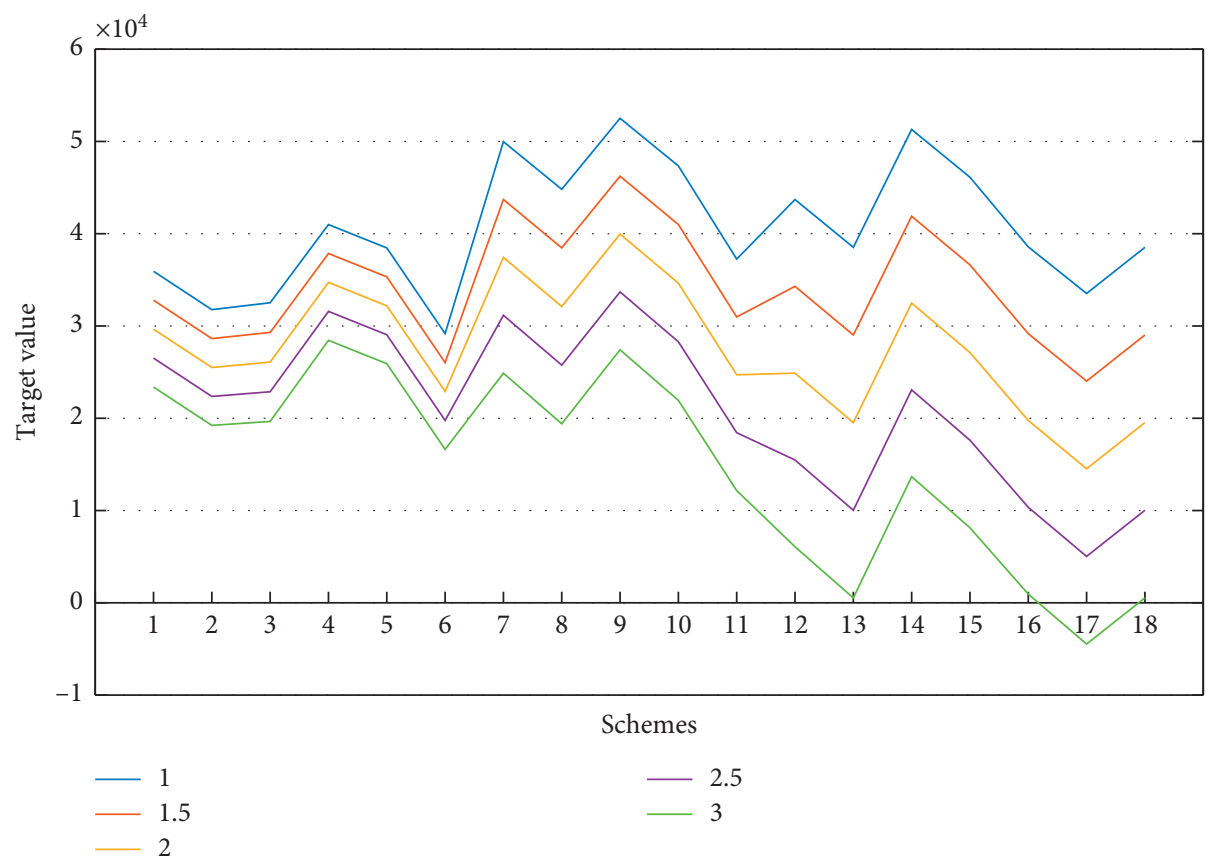

FiguRE 7: Costs of various schemes under different train operation cost coefficients.

values of schemes $\# 10, \# 15, \# 8, \# 13, \# 18, \# 17, \# 3$, and \#2 fluctuate greatly with the change of adjustment cost coefficient of existing trains, which indicates that the adjustment time of existing trains in these schemes is larger.

When the adjustment cost coefficient of the existing trains is relatively large, a scheme in which CT operation has less influence on existing trains should be selected. As shown in Figure 8, when the adjustment cost coefficient of existing train is 200 , the target value of scheme \#17 is larger than that of scheme \#4. With the increase of adjustment cost coefficient of existing trains, the target value of scheme \#17 is much lower than that of scheme \#4.

When the train operation cost coefficient $C_{z}=2$, the influence of different train adjustment cost coefficients on each scheme is shown in Figure 9. Regardless of the adjustment cost coefficient of the existing train, the model target value of scheme \#9 (train $2+$ train 5) is still the largest. Compared with $C_{z}=1$, the increase of the operation cost coefficient has a greater impact on schemes \#12-\#18, because these schemes divide 16 train units into three CTs. 


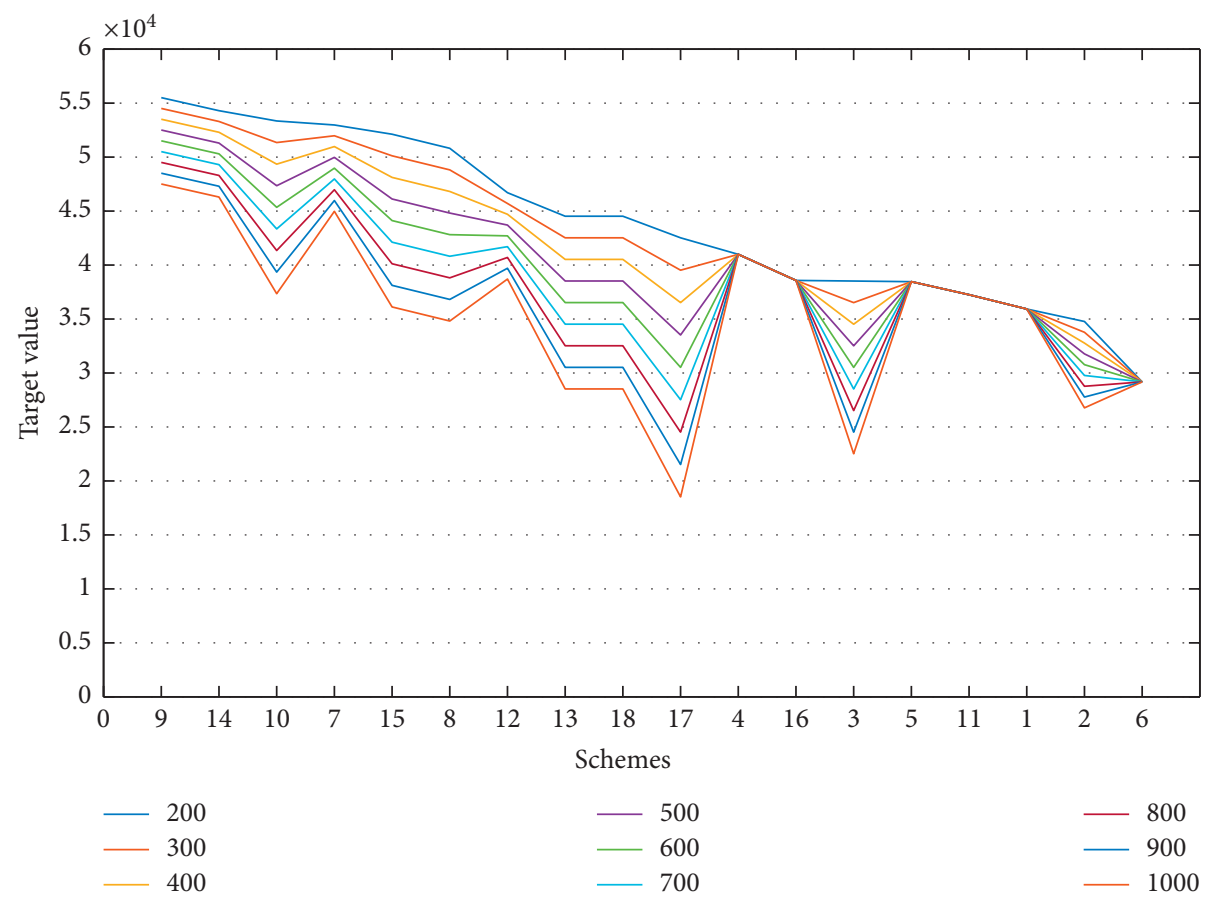

FiguRE 8: $C_{z}=1$ : target values of various schemes under different adjustment cost coefficients of existing railway trains.

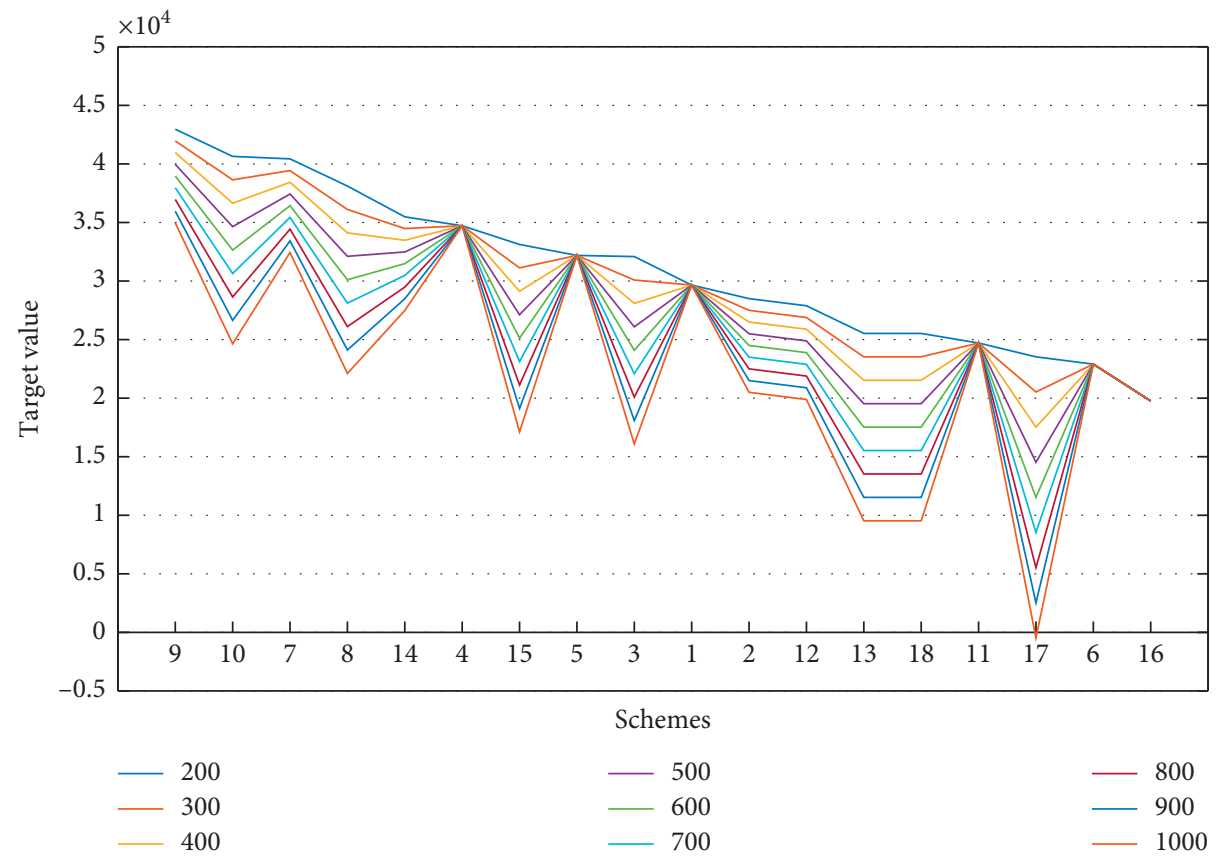

FIGURE 9: $C_{z}=2$ : target values of various schemes under different train adjustment cost coefficients of existing trains.

When the operation cost coefficient increases, the operation cost of enterprises will increase greatly.

When the train operation cost coefficient $C_{z}=3$, the influence of different train adjustment cost coefficients on each scheme is shown in Figure 10.

As shown in Figure 10, scheme \#9 is no longer the optimal scheme for any adjustment cost coefficient of the existing train. When the adjustment cost coefficients of the existing train are 200 and 300, scheme \#9 is still the best scheme, but when the adjustment cost coefficient is 400 or more, scheme \#4 becomes the best scheme. As shown in Figure 5, although scheme \#9 can attract more passengers than scheme \#4, the number of CTs in scheme \#9 is more than that in scheme \#4 under the same number of train units, the operation cost in scheme \#9 is higher than that in scheme \#4, and the impact of scheme \#9 on existing trains is greater than that of scheme \#4.

Since the adjustment cost coefficient of existing train is relatively high, the values of schemes \#15, \#13, \#18, \#17, and 


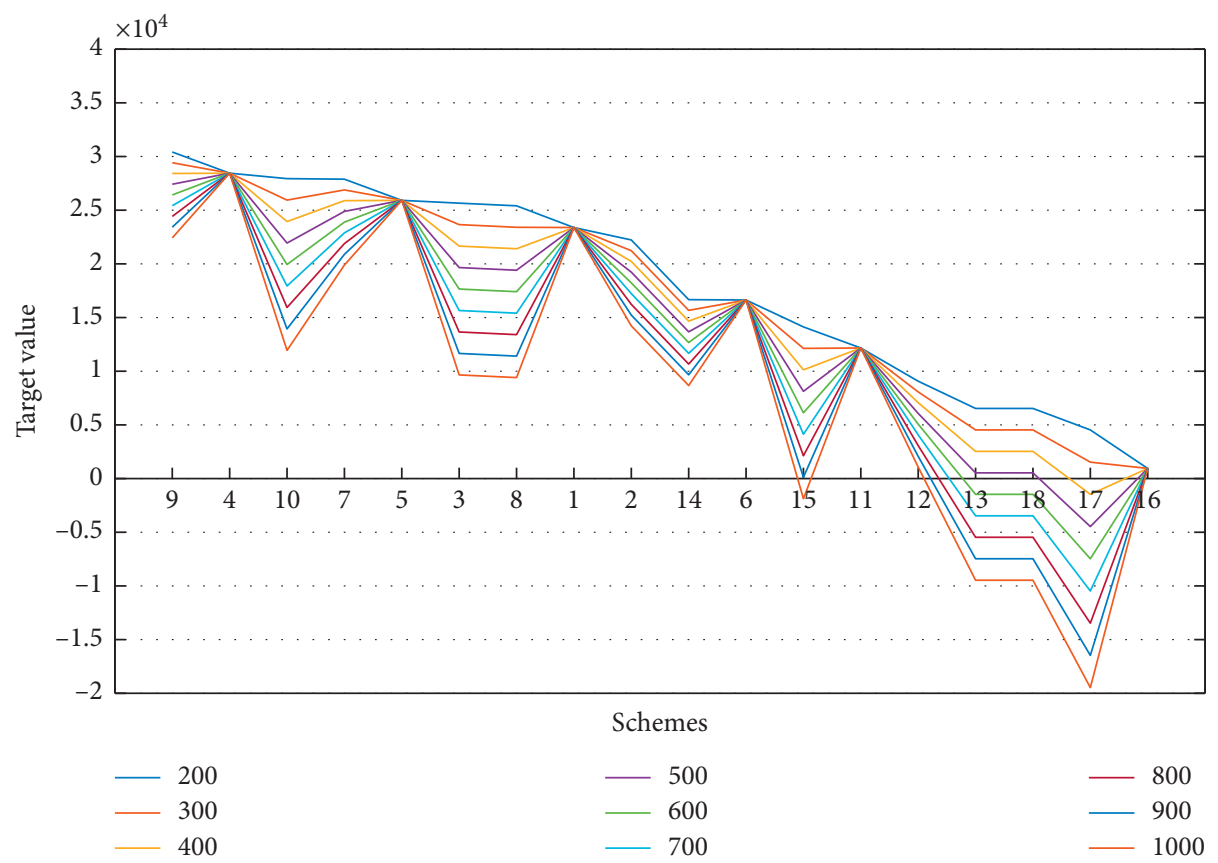

Figure 10: $C_{z}=3$ : target values of various schemes under different train adjustment cost coefficients of existing trains.

\#16 begin to become negative, and the corresponding operation scheme should not be selected. Therefore, when the cost coefficient of train operation is large, a small number of CTs are operated to get the greater benefits. If three CTs are selected, the target value of the model obtained by the enterprise will decrease.

\section{Conclusion}

Since many enterprises pay more and more attention to the development of demand-driven production and service mode, for passenger railway industries, CT operation mode may become a development mode of operation. The paper proposed a comprehensive method for the CT operation and adjustment. This method can be divided into three stages: demand acquisition, CT operation plan, and train operation adjustment on the existing timetable. The demand data are obtained through questionnaire survey, which can be used in next stage of CT operation plan. An alternative set of CTs is established through combining with the collected passenger travel wishes data and the existing timetable. Then, a bilevel programming model of train operation adjustment is established to maximize the profit of enterprises. Meanwhile, the adjustment method for the train conflict is established to resolve the conflict between CTs and existing trains, and the algorithm is built as the adjustment algorithm.

The innovation of the method in the paper is that a new train is inserted into the existing train to satisfy the passenger travel demand and get additional benefits to railway enterprise. For the model, the paper obtains the passenger demand for the CTs by fitting the previous questionnaire data; a bilevel programming model is established to maximize the revenue of enterprise and minimize the adjustment time cost of existing trains. Through the combination of multiple departure times for CTs, a variety of CT operation schemes can be obtained. The revenues of enterprise under the different train operation cost or different train adjustment cost of existing lines can be compared. The best CT operation plan under different conditions provides a strong support for railway enterprise to obtain the CT operation plan.

The paper only obtains passengers' travel intention through questionnaire survey, where data acquisition is slow and the data sources are limited. In the future, a particular APP for CT service is expected, and passengers can complete a series of services on this APP, such as choosing the expected route and time in the early stage, paying for the CT ticket online, and evaluating the service after the travel.

\section{Data Availability}

The data used to support this study are available from the corresponding author upon request.

\section{Disclosure}

The authors certify that they have participated sufficiently in the work to take public responsibility for the appropriateness of the experimental design and method and the collection, analysis, and interpretation of the data.

\section{Conflicts of Interest}

The authors declare that they have no conflicts of interest.

\section{Acknowledgments}

This work was supported by the Natural Science Foundation of Inner Mongolia (grant number: 2017BS0501), The "13th Five Year” Plan of Educational Science Research in Inner Mongolia (grant number: NGJGH2018016), the National 
Natural Science Foundation of China (grant number: 71971019), and National Key Research and Development Plan (grant number: 2018YFB1201420).

\section{References}

[1] M. Gallo, B. Montella, and L. D’Acierno, “The transit network design problem with elastic demand and internalisation of external costs: an application to rail frequency optimisation," Transportation Research Part C: Emerging Technologies, vol. 19, no. 6, pp. 1276-1305, 2011.

[2] L. D’Acierno, M. Gallo, B. Montella et al., "Analysis of the interaction between travel demand and rail capacity constraints," WIT Transactions on the Built Enviroment, vol. 128, pp. 197-207, 2012.

[3] L. D’Acierno, M. Gallo, B. Montella, and A. Placido, "Evaluation of travel demand impacts in the case of rail system failure," Procedia-Social and Behavioral Sciences, vol. 87, pp. 75-84, 2013.

[4] G. Feng, H. Chen, and M. Liu, "An ontology service model for flexible service customization," in Proceedings of the 2014 11th International Conference on Service Systems and Service Management (ICSSSM), Beijing, China, June 2014.

[5] T. Liu and A. Ceder, "Analysis of a new public-transportservice concept: customized bus in China," Transport Policy, vol. 39, pp. 63-76, 2015.

[6] J. H. Ma, F. Wang, J. Wang et al., "The study of customized bus site and route planning," Urban Public Transport, vol. 2, pp. 21-25, 2017.

[7] Y. Cao and J. Wang, "An optimization method of passenger assignment for customized bus," Mathematical Problems in Engineering, vol. 2017, Article ID 7914753, 9 pages, 2017.

[8] L. Tong, L. Zhou, J. Liu, and X. Zhou, "Customized bus service design for jointly optimizing passenger-to-vehicle assignment and vehicle routing," Transportation Research Part C: Emerging Technologies, vol. 85, pp. 451-475, 2017.

[9] J. Zhou, D. Z. W. Wang, and M. Meng, "Which service is better on a linear travel corridor: park \& ride or on-demand public bus?," Transportation Research Part A: Policy and Practice, vol. 118, pp. 803-818, 2018.

[10] J. Meng, Y. Cao, and Y. H. Wang, "Customized bus route vehicle schedule method cconsidering travel time windows," China Journal of Highway and Transport, vol. 31, pp. 143-150, 2018.

[11] J. Brake, J. D. Nelson, and S. Wright, "Demand responsive transport: towards the emergence of a new market segment," Journal of Transport Geography, vol. 12, no. 4, pp. 323-337, 2004.

[12] G. Cich, L. Knapen, S. Galland, J. Vuurstaek, and A. N. T. Bellemans, "Towards an agent-based model for demand-responsive transport serving thin flows," Procedia Computer Science, vol. 83, pp. 952-957, 2016.

[13] J. Haverkamp, "Demand responsive rail transport," M. A thesis, 2017.

[14] C. F. Daganzo, "Checkpoint dial-a-ride systems," Transportation Research Part B: Methodological, vol. 18, no. 4-5, pp. 315-327, 1984.

[15] A. Pratelli, "Some approaches on checkpoint dial-a-ride problems," in Urban Transport VIII, pp. 131-140, WIT Press, Billerica, MA, USA, 2002.

[16] H. B. Zhang and B. T. Dong, "Railway timetable saturation based on capacity calculation," Journal of Transportation Systems Engineering and Information Technology, vol. 11, pp. 129-134, 2011.

[17] A. Lulli, L. Oneto, R. Canepa et al., "Large-scale railway networks train movements: a dynamic, interpretable, and robust hybrid data analytics system," in Proceedings of the EEE 5th International Conference on Data Science and Advanced Analytics (DSAA), Turin, Italy, October 2018.

[18] L. Oneto, I. Buselli, A. Lulli, S. Canepa, and D. Anguita, "A dynamic, interpretable, and robust hybrid data analytics system for train movements in large-scale railway networks," International Journal of Data Science and Analytics, 2019.

[19] UIC International Union of Railways, UIC Leaflet 406, Capacity, UIC International Union of Railways, aris, France, 2013.

[20] J. L. Espinosa-Aranda and R. García-Ródenas, "A demandbased weighted train delay approach for rescheduling railway networks in real time," Journal of Rail Transport Planning \& Management, vol. 3, no. 1-2, pp. 1-13, 2013.

[21] F. Corman, A. D'Ariano, A. D. Marra, and M. Pacciarelli, "Integrating train scheduling and delay management in realtime railway traffic control," Transportation Research Part E: Logistics and Transportation Review, vol. 105, pp. 213-239, 2017.

[22] S. Zhan, L. G. Kroon, L. P. Veelenturf, and J. C. Wagenaar, "Real-time high-speed train rescheduling in case of a complete blockage," Transportation Research Part B: Methodological, vol. 78, pp. 182-201, 2015.

[23] G. Wagenaar, M. Dotoli, N. Epicoco, and C. Seatzu, "A decision making procedure for robust train rescheduling based on mixed integer linear programming and data envelopment analysis," Applied Mathematical Modelling, vol. 52, pp. 255-273, 2017.

[24] L. Seatzu, E. Fumeo, G. Clerico et al., "Train delay prediction systems: a big data analytics perspective," Big Data Research, vol. 11, pp. 54-64, 2018.

[25] R. M. P. Goverde, "A delay propagation algorithm for largescale railway traffic networks," Transportation Research Part C: Emerging Technologies, vol. 18, no. 3, pp. 269-287, 2010.

[26] R. M. P. Goverde, B. Heidergott, and G. Merlet, "A coupling approach to estimating the Lyapunov exponent of stochastic max-plus linear systems," European Journal of Operational Research, vol. 210, no. 2, pp. 249-257, 2011.

[27] X. J. Li, B. M. Han, D. W. Li et al., "Method for high-speed train operation adjustment based on switching Max-PlusLinear System and Ordinal Optimization," China Railway Science, vol. 34, pp. 124-130, 2013.

[28] X. J. Li, Y. Y. Huo, Z. Y. Yan et al., "An optimization method for operation adjustment of high-speed delayed trains," Discrete Dynamics in Nature and Society, vol. 2019, Article ID 9846970, 16 pages, 2019.

[29] W. L. Zhou, L. Y. Qu, F. Shi et al., "Train scheduling on highspeed rail network based on fixed order optimization," Journal of Railway Science and Engineering, vol. 15, pp. 551-558, 2018.

[30] N. Ghaemi, A. A. Zilko, F. Yan, O. Cats, D. Kurowicka, and R. M. P. Goverde, "Impact of railway disruption predictions and rescheduling on passenger delays," Journal of Rail Transport Planning \& Management, vol. 8, no. 2, pp. 103-122, 2018.

[31] L. D’Acierno, M. Botte, M. Gallo, and B. Montella, "Defining reserve times for metro systems: an analytical approach," Journal of Advanced Transportation, vol. 2018, Article ID 5983250, 15 pages, 2018.

[32] L. D’Acierno, M. Botte, A. Placido, C. Caropreso, and B. Montella, "Methodology for determining dwell times consistent with passenger flows in the case of metro services," Urban Rail Transit, vol. 3, no. 2, pp. 73-89, 2017.

[33] Y. J. Wang and X. Y. Yin, "Research the simulation model of the passenger travel behavior in urban rail platform," in Proceedings of the AIP Conference, Bikaner, India, November 2017.

[34] M. Botte and L. D’Acierno, "Dispatching and rescheduling tasks and their interactions with travel demand and the energy domain: models and algorithms," Urban Rail Transit, vol. 4, no. 4, pp. 163-197, 2018. 